\title{
Ontogeny and morphological diversity in immature mites: Preface to Part V with a summary of contributions so far
}

\author{
ZHI-QIANG ZHANG ${ }^{1,2}$ \\ ${ }^{1}$ Landcare Research, Private Bag 92170, Auckland, New Zealand. \\ ”zhangZ@landcareresearch.co.nz @ https://orcid.org/0000-0003-4172-0592 \\ ${ }^{2}$ School of Biological Sciences, Centrefor Biodiversity \& Biosecurity, The University of Auckland, Auckland, New Zealand
}

It is my great pleasure to present to you the fifth part in this series of special volumes on mite ontogeny and morphological diversity (Zhang et al. 2018, 2019, 1920a,b). Despite the global COVID-19 pandemic, our ontogeny enthusiasts managed to continue their research and contributed eight papers to this volume, with one paper on Mesostigmata (Zhang et al. 2021), five papers on Oribatida (Bayartogtokh et al. 2021a,b; Seniczak, S., Ivan, Marquardt \& Seniczak, A. 2021a,b; Seniczak, A. \& Seniczak, S. 2021) and two papers on Trombidiformes (Negm \& Gotoh 2021; Xu \& Zhang 2021).

Prior to the start of this series, two journals (Systematic \& Applied Acarology; Zootaxa) each published about 28\% of the mite ontogenetic papers from 2015 to 2017 (Liu \& Zhang 2018). Since December 2018, five volumes with a total of 47 papers in 1,077 pages were published in this series in just 36 months, and 41 of these reported on mite ontogeny (Table 1); in comparison, Systematic \& Applied Acarology published only 22 papers on mite morphological ontogeny during the same period. Among the 41 papers on morphological ontogeny in these five volumes in Zootaxa, 20 papers (or 48.8\%) are on Sarcoptiformes, 15 (or 34.1\%) are on Trombidiformes, and only 7 (17.1\%) are on Mesostgimata (Table 1); these papers reported on morphological ontogenies of 113 species. Among the 18 papers on oribatid ontogenies, the team of Stanisław Seniczak and Anna Seniczak (plus other collaborators sometimes) co-authored 12 (or 66.7\%) (Table 1). It is commendable that this team also co-authored 10 of 15 papers on mite ontogenies in Systematic \& Applied Acarology in 2018 (Zhang 2019), and 12 of 22 papers from 2019 to 2021 (Seniczak, A., Seniczak, S. \& Jordal 2019; Seniczak, A. \& Seniczak, S. 2019, 2020a,b,c; 2021; Seniczak, S., Ivan, Kaczmarek \& Seniczak, A. 2020, 2021; Seniczak, S., Ivan \& Seniczak, A. 2019; 2020; Seniczak, S., Kaczmarek \& Seniczak, A. 2021; Seniczak, S., Seniczak, A., Kaczmarek, Marquardt \& Jangazieva 2020).

TABLE 1. The numbers of papers (species) in the five special volumes on "Ontogeny and morphological diversity in immature mites" published in Zootaxa from 2018 to 2021.

\begin{tabular}{|c|c|c|c|c|c|c|c|}
\hline \multirow[t]{2}{*}{ Parts } & \multirow[t]{2}{*}{ Mesostigmata } & \multicolumn{2}{|c|}{ Sarcoptiformes } & \multirow[t]{2}{*}{ Trombidiformes } & \multirow[t]{2}{*}{ Others } & \multirow[t]{2}{*}{ Total } & \multirow[t]{2}{*}{ References } \\
\hline & & Oribatida & Astigmata & & & & \\
\hline I & $2(2)$ & $3(3)$ & 0 & $4(5)$ & 2 & $11(10)$ & Zhang et al. (2018) \\
\hline II & $1(1)$ & $5(5)$ & 0 & $3(6)$ & 1 & $10(12)$ & Zhang et al. (2019) \\
\hline III & $2(3)$ & $3(3)$ & $1(1)$ & $2(2)$ & 1 & $9(9)$ & Zhang et al. (2020b) \\
\hline IV & $1(1)$ & $2(2)$ & $1(1)$ & $3(70)$ & 1 & $8(74)$ & Zhang et al. (2020a) \\
\hline $\mathrm{V}$ & $1(1)$ & $5(5)$ & 0 & $2(2)$ & 1 & $9(8)$ & Fuangarworn et al. (2021) \\
\hline Total & $7(8)$ & $18(18)$ & $2(2)$ & $14(85)$ & 6 & $47(113)$ & \\
\hline
\end{tabular}

Due to the continued interest in this series, we will publish part 6 in mid-2022. Those who are interested in submitting papers should contact me for more information.

I am grateful my co-editors Dr Marut Fuangarworn (Thailand) and Mr Alihan Katlav (Australia), as well as our reviewers and authors for their contributions, and Lilian Zhang (New Zealand) for reading the draft of this manuscript. 


\section{References}

Bayartogtokh, B., Ermilov, S.G., Shtanchaeva, U.Y. \& Subías, L.S. (2021a) Ontogenetic instars of Hermannia longisetosa Subías \& Shtanchaeva, 2013, with remarks on juveniles of Hermanniidae (Acari: Oribatida). Zootaxa, 5086 (1), 49-68. https://doi.org/10.11646/zootaxa.5086.1.6

Bayartogtokh, B., Ermilov, S.G., Shtanchaeva, U.Y. \& Subías, L.S. (2021b) Ontogenetic instars of Melanozetes paramollicomus sp. nov., with remarks on morphological ontogeny of Sphaerozetinae (Acari: Oribatida: Ceratozetidae). Zootaxa, 5086 (1), 69-89.

https://doi.org/10.11646/zootaxa.5086.1.7

Fuangarworn, M., Zhang, Z.-Q. \& Katlav, A. (2021) Ontogeny and morphological diversity in immature mites (Part V). Zootaxa, 5086, 001-173.

https://doi.org/10.11646/zootaxa.5086.1

Liu, J.-F. \& Zhang, Z.-Q. (2018) A survey of descriptions of immature instars of mites during the last three years. Zootaxa, 4540 (1), 211-224. https://doi.org/10.11646/zootaxa.4540.1.13

Negm, M.W. \& Gotoh, T. (2021) Redescription of Panonychus caglei Mellott, 1968, with ontogenetic development (Acari: Tetranychidae). Zootaxa, 5086 (1), 157-173. https://doi.org/10.11646/zootaxa.5086.1.11

Seniczak, A., Seniczak, S. \& Jordal, B.H. (2019) Molecular and ontogeny studies clarify systematic status of Chamobates borealis (Acari, Oribatida, Chamobatidae): an integrated taxonomy approach. Systematic \& Applied Acarology, 24 (12), 2409-2426. https://doi.org/10.11158/saa.24.12.9

Seniczak, A. \& Seniczak, S. (2019) Morphological ontogeny of Caleremaeus monilipes (Acari: Oribatida: Caleremaeidae), with comments on Caleremaeus Berlese. Systematic \& Applied Acarology, 24 (11), 1995-2009. https://doi.org/10.11158/saa.24.11.3

Seniczak, A. \& Seniczak, S. (2020a) Morphological ontogeny of Amerus polonicus (Acari: Oribatida: Ameridae), and comments on Amerus Berlese. Systematic \& Applied Acarology, 25 (10), 1840-1856. https://doi.org/10.11158/saa.25.10.8

Seniczak, A. \& Seniczak, S. (2020b) Morphological ontogeny of Fuscozetes coulsoni sp. nov. (Acari: Oribatida: Ceratozetidae) from Svalbard, Norway. Systematic \& Applied Acarology, 25 (4), 680-696. https://doi.org/10.11158/saa.25.4.8

Seniczak, A. \& Seniczak, S. (2020c) Morphological ontogeny of Limnozetes solhoyorum sp. nov. (Acari: Oribatida: Limnozetidae) from Norway, with comments on Limnozetes Hull. Systematic \& Applied Acarology, 25 (2), 327-348. https://doi.org/10.11158/saa.25.2.10

Seniczak, A. \& Seniczak, S. (2021) Morphological ontogeny of Limnozetes schatzi sp. nov. (Acari: Oribatida: Limnozetidae) from Norway. Systematic \& Applied Acarology, 25 (10), 1974-1991. https://doi.org/10.11158/saa.26.10.10

Seniczak, A. \& Seniczak, S. (2021) Morphological ontogeny and ecology of Ceratozetes parvulus (Acari: Oribatida: Ceratozetidae). Zootaxa, 5086 (1), 111-134. https://doi.org/10.11646/zootaxa.5086.1.9

Seniczak, S., Ivan, O., Kaczmarek, S. \& Seniczak, A. (2020) Morphological ontogeny of Lopheremaeus mirabilis (Acari: Oribatida: Plateremaeidae), and comments on Lopheremaeus Paschoal. Systematic \& Applied Acarology, 25 (12), $2147-2164$. https://doi.org/10.11158/saa.25.12.1

Seniczak, S., Ivan, O., Kaczmarek, S. \& Seniczak, A. \& (2021) Morphological ontogeny of Eremobelba geographica (Acari: Oribatida: Eremobelbidae), with comments on Eremobelba Berlese. Systematic \& Applied Acarology, 25 (4), $749-768$. https://doi.org/10.11158/saa.26.4.7

Seniczak, S., Ivan, O., Marquardt, T. \& Seniczak, A. (2021a) Morphological ontogeny of Oribatella similesuperbula (Acari: Oribatida: Oribatellidae). Zootaxa, 5086 (1), 90-110. https://doi.org/10.11646/zootaxa.5086.1.8

Seniczak, S., Ivan, O., Marquardt, T. \& Seniczak, A. (2021b) Morphological ontogeny of Perlohmannia nasuta (Acari, Oribatida, Perlohmanniidae), with comments on Perlohmannia Berlese. Zootaxa, 5086 (1), 29-48. https://doi.org/10.11646/zootaxa.5086.1.5

Seniczak, S., Ivan, O. \& Seniczak, A. (2019) Morphological ontogeny of Tectoribates ornatus (Acari: Oribatida: Tegoribatidae), with comments on Tectoribates Berlese. Systematic \& Applied Acarology, 24 (12), 2290-2310. https://doi.org/10.11158/saa.24.12.2

Seniczak, S., Ivan, O. \& Seniczak, A. (2020) Morphological ontogeny of Damaeolus ornatissimus (Acari: Oribatida: Damaeolidae), with comments on Damaeolus Paoli. Systematic \& Applied Acarology, 25 (3), 459-478. https://doi.org/10.11158/saa.25.3.7

Seniczak, S., Kaczmarek, S. \& Seniczak, A. (2021) Morphological ontogeny of Proteremaeus oralensis sp. nov. (Acari: Oribatida: Eremaeidae) from Kazakhstan, and comments on Proteremaeus Piffl. Systematic \& Applied Acarology, 25 (5), $902-$ 917. 
https://doi.org/10.11158/saa.26.5.6

Seniczak, S., Seniczak, A., Kaczmarek, S., Marquardt, T. \& Jangazieva, B. (2020) Morphological ontogeny of Cosmochthonius oralensis sp. nov. (Acari: Oribatida: Cosmochthonidae) from Kazakhstan, and comments on Cosmochthonius Berlese. Systematic \& Applied Acarology, 25 (1), 31-50. https://doi.org/10.11158/saa.25.1.3

Xu, Y. \& Zhang, F.-P. (2021) A new species of the genus Colopalpus Pritchard and Baker (Trombidiformes: Tenuipalpidae) from China, with ontogenetic patterns in chaetotaxy. Zootaxa, 5086 (1), 135-156. https://doi.org/10.11646/zootaxa.5086.1.10

Zhang, B., Ma, M. \& Fan, Q.-H. (2021) Morphological ontogeny of Neoseiulus zwoelferi (Acari: Phytoseiidae). Zootaxa, 5086 (1), 7-28.

https://doi.org/10.11646/zootaxa.5086.1.4

Zhang, Z.-Q. (2019) Introducing the second volume on the ontogeny and morphological diversity in immature mites. Zootaxa, $4717(1), 5-6$. https://doi.org/10.11646/zootaxa.4717.1.3

Zhang, Z.-Q., Fuangarworn, M., Fan, Q.-H. \& Yi, T.-C. (Eds) (2020a) Ontogeny and morphological diversity in immature mites (Part IV). Zootaxa, 4900 (1), 1-200. https://doi.org/10.11646/zootaxa.4900.1

Zhang, Z.-Q., Fuangarworn, M. \& Seeman, O.D. (Eds) (2020b) Ontogeny and morphological diversity in immature mites (Part III). Zootaxa, 4857 (1), 1-250. https://doi.org/10.11646/zootaxa.4857.1

Zhang, Z.-Q., Fuangarworn, M., Seeman, O. \& Mironov, S. (Eds) (2019) Ontogeny and morphological diversity in immature mites (Part II). Zootaxa, 4717 (1), 1-230. https://doi.org/10.11646/zootaxa.4717.1

Zhang, Z.-Q., Seeman, O., Fuangarworn, M. \& Fan, Q.-H. (Eds) (2018) Ontogeny and morphological diversity in immature mites (Part I). Zootaxa, 4540 (1), 1-224.

https://doi.org/10.11646/zootaxa.4540.1 